\title{
Analyse des essais industriels des turbines-pompes hors des zones de fonctionnement normal et lors des transitoires
}

\author{
Analysis of industrial testing of turbine pumps \\ outside normal and during transient \\ operating conditions
}

\author{
S. Casacci et P. Jarriand
}

Neyrpic Grenoble

\section{Généralités}

L'exploitation des centrales d'accumulation impose quotidiennement de nombreux démarrages et arrêts aux turbines-pompes. Ces machines fonctionnent beaucoup plus fréquemment que les turbines conventionnelles hors de leur domaine optimal d'utilisation. Lors des régimes transitoires consécutifs à des disjonctions, elles se trouvent soumises à des sollicitations très élevées.

L'évolution des turbines-pompes monocellulaires nécessite :

- un contrôle rigoureux, sur prototype, des hypothèses retenues lors du dimensionnement de la machine ;

- et la connaissance précise des charges appliquées aux différents organes dans tous les régimes de fonctionnement, permanent ou transitoire.

Nous nous proposons de présenter ici un certain nombre de résultats de mesures effectuées sur un des deux groupes équipant la centrale du CHEYLAS, et concernant plus particulièrement les régimes transitoires en pompe et en turbine.

Cet aménagement, réalisé par EDF, constitue la dernière installation de l'équipement de la vallée de l'Arc. Le bassin supérieur, créé par la construction d'une digue, a une capacité utile de $4,7 \mathrm{~km}^{3}$ entre les cotes 491 et $499 \mathrm{~m}$. Il reçoit un apport gravitaire d'environ $63 \mathrm{~m}^{3} / \mathrm{sec}$. en provenance du bassin de compensation de Longefan, par l'intermédiaire de la galerie de Belledonne. Une galerie en charge de $3900 \mathrm{~m}$ de longueur et de $7,5 \mathrm{~m}$ de diamètre, munie d'une vanne de tête, relie ce bassin à une cheminée d'équilibre à diaphragme.

La conduite forcée en puits blindé de $5,60 \mathrm{~m}$ de diamètre et de $500 \mathrm{~m}$ de longueur se subdivise en 2 rameaux de $4 \mathrm{~m}$ de diamètre et de $120 \mathrm{~m}$ de longueur, alimentant chacun un groupe.

Les groupes et leur robinet sphérique de garde sont installés dans 2 puits de $19,60 \mathrm{~m}$ de diamètre et de $66 \mathrm{~m}$ de profondeur. Les aspirateurs des groupes sont reliés à une conduite de restitution de $760 \mathrm{~m}$ de longueur par l'intermédiaire d'un bassin d'expansion d'une capacité utile de $10000 \mathrm{~m}^{3}$. Chaque groupe peut être isolé des ouvrages basse pression par une vanne wagon.

\section{Caractéristiques principales des machines}

Du type monoétage à distributeur mobile, elles comportent un arbre d'axe vertical, guidé par trois paliers. Le pivot placé sous l'alternateur repose sur une dalle en béton. Le démontage de la turbinepompe s'effectue à l'étage intermédiaire. Le démarrage en pompe, roue dénoyée, est réalisé en asynchrone avec interposition temporaire de réactances. Les caractéristiques de ces machines sont résumées dans le Tableau I.

\section{Tableau I - Caractéristiques principales des machines} de la centrale du CHEYLAS

\begin{tabular}{|c|c|c|c|c|}
\hline & \multicolumn{2}{|c|}{ Pompe } & \multicolumn{2}{|c|}{ Turbine } \\
\hline $\begin{array}{l}\text { Chute nette ou hauteur } \\
\text { d'élévation }(\mathrm{m})\end{array}$ & 267,55 & 247,8 & 256,2 & \\
\hline Débit $\left(\mathrm{m}^{3} / \mathrm{sec}.\right)$ & 79,5 & 89,1 & 114 & 107,4 \\
\hline Puissance (MW) & 229,3 & 236,7 & 252 & 214 \\
\hline $\begin{array}{l}\text { Vitesse de rotation } \\
\text { (tr } / \mathrm{min})\end{array}$ & \multicolumn{4}{|c|}{300} \\
\hline $\begin{array}{l}\text { Enfoncement de la } \\
\text { roue }(m)\end{array}$ & \multicolumn{4}{|c|}{29} \\
\hline Diamètre de roue $(\mathrm{mm})$ & \multicolumn{4}{|c|}{4780} \\
\hline
\end{tabular}




\section{Mesures effectuées sur une turbine-pompe}

Parmi les nombreuses mesures effectuées dans le but :

- de contrôler le comportement des organes de vannage, de la ligne d'arbres, des paliers et du pivot:

- d'observer l'évolution des pressions en différents points du conduit hydraulique ;

nous analyserons plus particulièrement celles qui se rapportent aux fluctuations des paramètres suivants :

- couple sur l'arbre moteur

- couple sur un tourillon de directrice

- poussée radiale sur le palier turbine

- poussée axiale

et pour certains fonctionnements :

- pression aspirateur

$C_{A}$
$C_{T}$

$C_{T}$

$P_{R}$

$P_{A X}$

$P_{A S}$

La figure 1 représente l'emplacement des points de mesure correspondants.

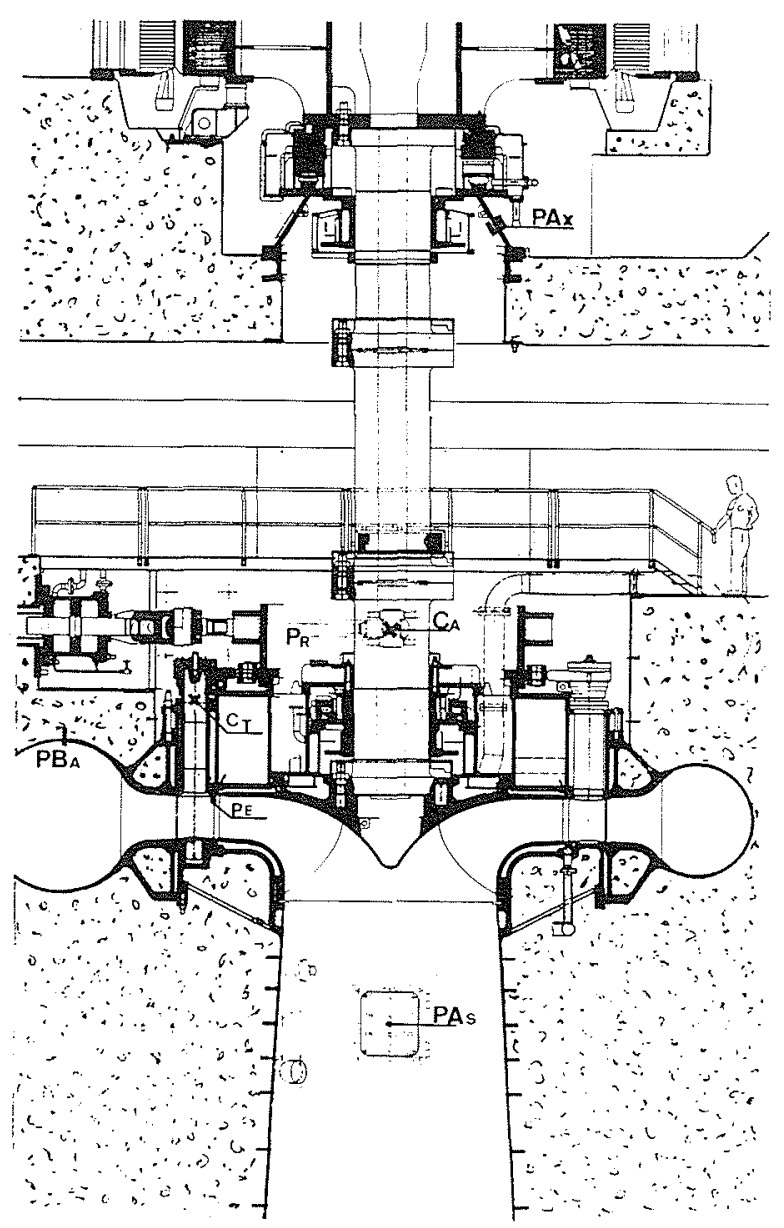

Figure 1 - Implantation des points de mesure sur une turbinepompe du Cheylas.

L'analyse effectuée dans cette communication se limitera aux fonctionnements précisés ci-dessous, à savoir :

- Fonctionnement en turbine :

- prise de charge ;

- déclenchement à pleine charge ;
- déclenchement à pleine charge, vannage bloqué ouvert, fermeture par le robinet sphérique ;

- coupure en charge par le robinet sphérique, le groupe restant couplé sur le réseau.

- Fonctionnement en pompe :

- prise de débit en pompe ;

- déclenchement à pleine ouverture ;

- déclenchement avec vannage bloqué ouvert et coupure du débit par le robinet sphérique.

\section{Fréquences caractéristiques}

Nous indiquons ci-après quelques unes des fréquences caractéristiques qui pourront apparaître sur les enregistrements au cours des différents essais:

- fréquence de rotation

$$
f_{0}=\frac{n}{60}=5 \mathrm{~Hz}
$$

- fréquence d'excitation par les aubes de la roue

$$
f_{R}=\frac{n Z R}{60}=35 \mathrm{~Hz}=7 f_{0}
$$

- fréquence d'excitation due aux sillages des directrices

$$
f_{D}=\frac{n Z D}{60}=100 \mathrm{~Hz}=20 f_{0}
$$

- fréquence propre de torsion de l'arbre

$$
f_{p A}=14 \mathrm{~Hz}
$$

- fréquence propre de torsion d'une directrice dans l'eau

$$
f_{p_{1} D}=150 \mathrm{~Hz}
$$

\section{Essais en turbine}

\section{Prise de charge}

La figure 2 représente l'évolution de l'amplitude des fluctuations, crête à crête, de différents paramètres caractéristiques en fonction de la puissance fournie par la turbine. Pour des charges supérieures à $40 \%$ de la puissance maximale, l'amplitude des fluctuations reste faible et le fonctionnement de la machine est particulièrement calme.

Au-dessous de cette puissance, elles croissent rapidement pour atteindre leur maximum en marche à vide, où l'on note en particulier des fluctuations $\mathrm{du}$ couple moteur atteignant $\pm 15 \%$ du couple nominal et des poussées radiales de $\pm 70 \mathrm{~T}$ sur le palier turbine. Ces fluctuations, dues aux conditions d'alimentation de la roue, sur les aubes de laquelle peuvent se produire des décollements instables, ont pour fréquences caractéristiques:

- la fréquence propre de torsion de l'arbre, pour le couple moteur $C_{A}$; 


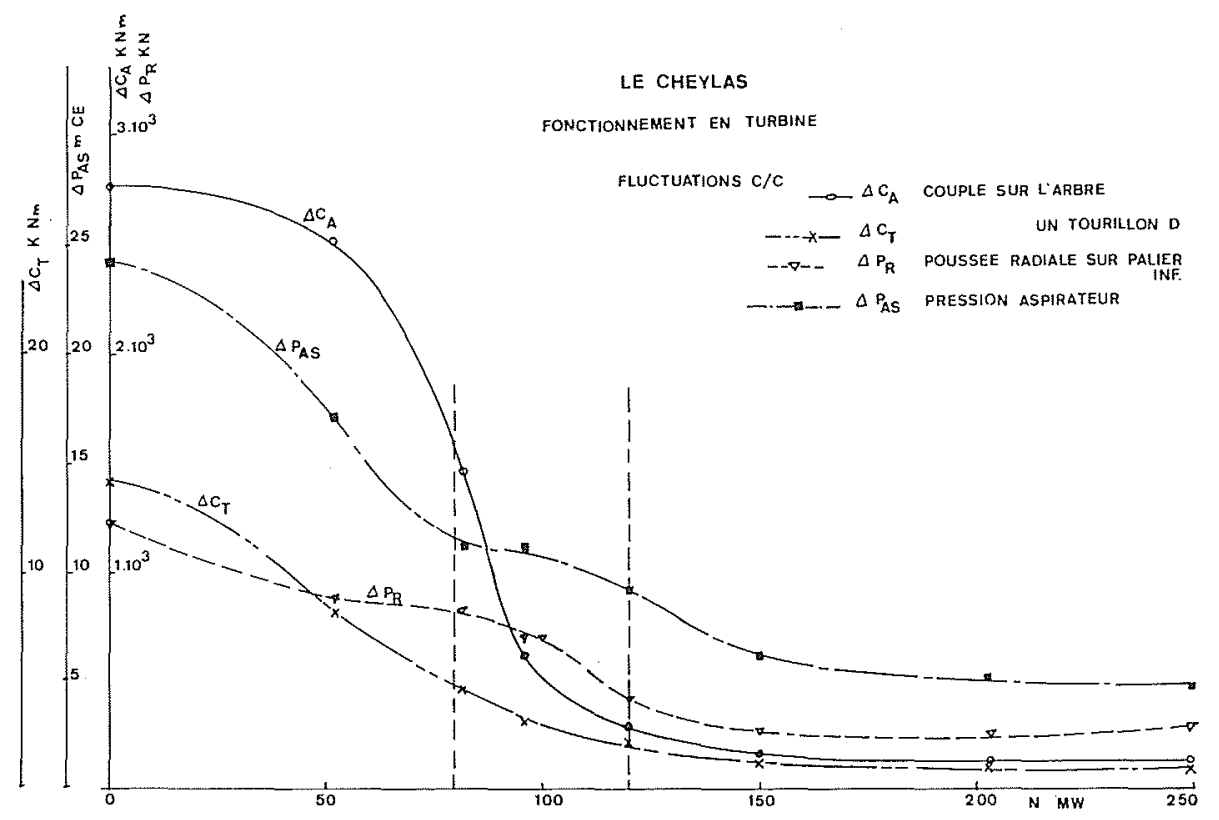

Figure 2 - Evolution de l'amplitude des fluctuations (crête à crête) des différents paramètres en fonction de la puissance fournie par la turbine.

- la fréquence de rotation, pour les poussées radiales $P_{R}$;

- la fréquence de passage des aubes de la roue et ses 2 premiers harmoniques, pour le couple sur les directrices $C_{T}$.

Ces pulsations du couple au voisinage de la marche à vide apparaissent lorsque la roue cesse de fonctionner en turbine à action et commence à travailler comme une machine à réaction. De nombreux essais montrent que ce type de fluctuations augmente très sensiblement lorsque la vitesse tangentielle, au droit de la roue, dépasse $\sqrt{(2 \mathrm{hg})}$. Ces variations du couple moteur au voisinage du synchronisme peuvent compromettre le réglage de la vitesse dans cette zone et rendre très difficile le couplage. L'analyse spectrale montre un seul pic très accusé pour la fréquence propre de torsion de la ligne d'arbre qui semble être excitée par des impulsions de fréquences plus basses liées à la structure de l'écoulement dans la roue.

Les fonctionnements à charge partielle peuvent être assez contraignants surtout s'ils sont de longue durée et il semble préférable de ne pas exploiter les turbines-pompes en-dessous de $40 \%$ de leur puissance nominale.

\section{Déclenchement de pleine charge}

\section{Déclenchement normal d'un groupe (Fig. 3)}

Cette figure reproduit l'évolution des fluctuations des couples sur l'arbre moteur $\Delta C_{T}$ et sur le tourillon d'une directrice $\Delta C_{A}$, ainsi que des poussées radiales $\Delta D_{R}$, tirées de l'enregistrement d'un déclenchement à pleine charge $(250 \mathrm{MW})$. On observe une augmentation très rapide des fluctuations avec la vitesse de rotation. Elles atteignent leurs valeurs maximales au voisinage de la survitesse maximale. Elles décroissent ensuite assez rapidement et passent par un minimum dans le quadrant pompe à rotation inverse.
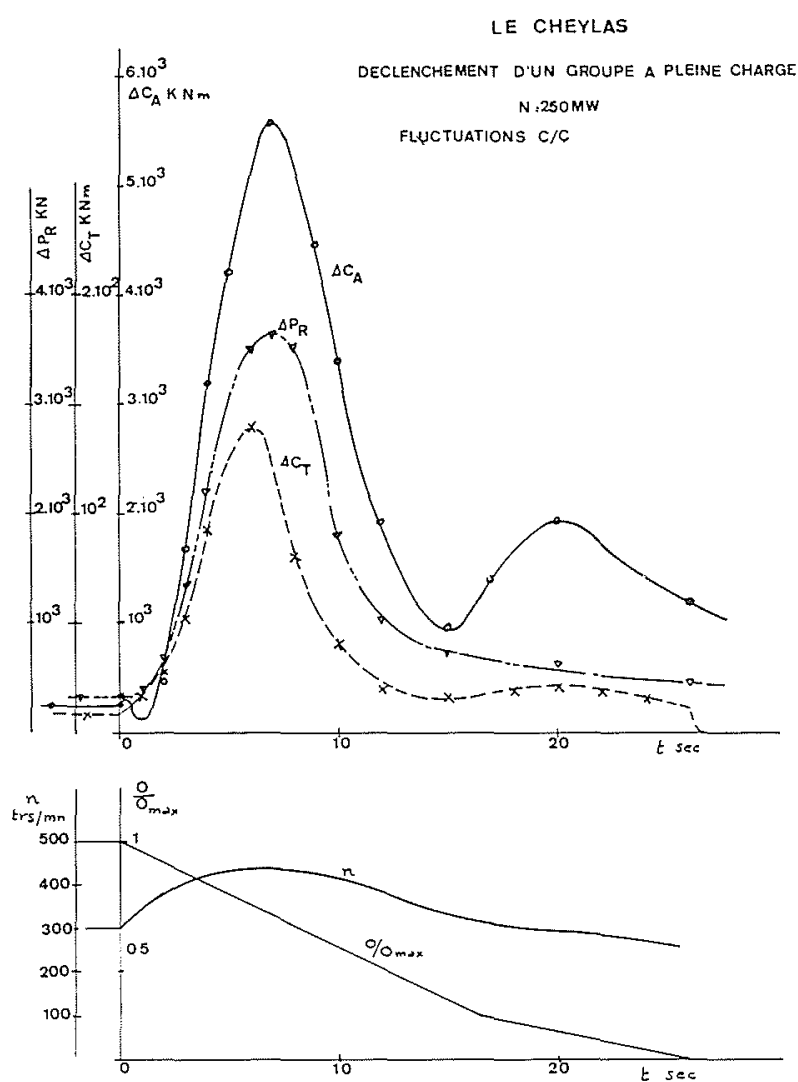

Figure 3 - Evolution des fluctuations, en fonction de la vitesse au rotation:

$\longrightarrow$ du couple sur l'arbre moteur $\Delta C_{A}$;

$\ldots-\forall \ldots$ du couple sur le tourillon d'une directrice $\Delta C_{T}$; - $-\mathrm{X}$ - - des penssées radiales $\Delta P_{e}$ 
Les valeurs extrêmes des sollicitations s'élèvent à : - $\pm 280 \mathrm{Tm}$, soit $\pm 35 \%$ du couple nominal sur l'arbre moteur, la fréquence de $14 \mathrm{~Hz}$ constituant le fondamental

$- \pm 7 \mathrm{Tm}$ sur le tourillon des directrices, le couple moyen étant pratiquement nul. On note ici encore la fréquence de passage des aubes de la roue et ses deux premiers harmoniques;

$- \pm 180 \mathrm{~T}$ sur le palier turbine. L'analyse spectrale met en évidence deux pics caractéristiques à 4,2 et $12 \mathrm{~Hz}$; on n'observe pas de sollicitations à la fréquence de rotation.

Ces amplitudes sont à comparer avec celles qui se produisent en marche normale et qui atteignent :

- \pm 6 Tm sur l'arbre moteur;

- $\pm 0,4 \mathrm{Tm}$ sur le tourillon de directrice;

$- \pm 14 \mathrm{~T}$ sur le palier turbine.

\section{Déclenchement avec vannage bioqué ouvert}

La figure 4 reproduit l'enregistrement de cet essai effectué en maintenant volontairement le vannage à pleine ouverture durant la manceuvre de fermeture du robinet sphérique. L'autre groupe déclenché simultanément fermait normalement. Cet essai correspond au cas le plus contraignant des régimes transitoires en turbine. Les courbes de la figure 5, tirées de cet enregistrement, montrent l'évolution des mêmes paramètres que précédemment, au cours de ce fonctionnement. On observe des évolutions analogues de la vitesse et des fluctuations, celles-ci atteignant toutefois des valeurs plus élevées que lors d'un déclenchement normal. Les couples pulsatoires s'élèvent à :

- $\pm 360 \mathrm{Tm}\left( \pm 45 \%\right.$ de $\left.C_{n}\right)$ sur l'arbre turbine ;

-. $\pm 9 \mathrm{Tm}$ sur les tourillons de directrices;

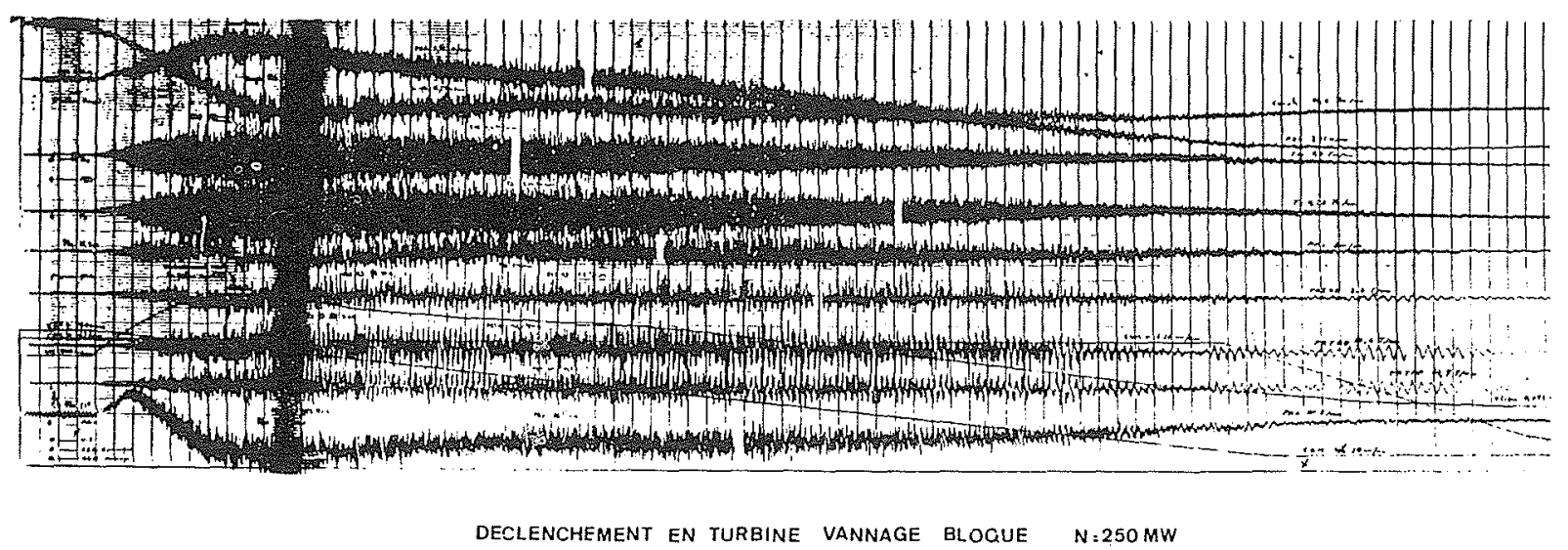

\section{LE CHEYLAS}

Figu:e 4 - Diagramme de déclenchement en turbine avec vannage bloqué à pleine ouverture durant la manœuvre de fermeture du robinet sphérique.

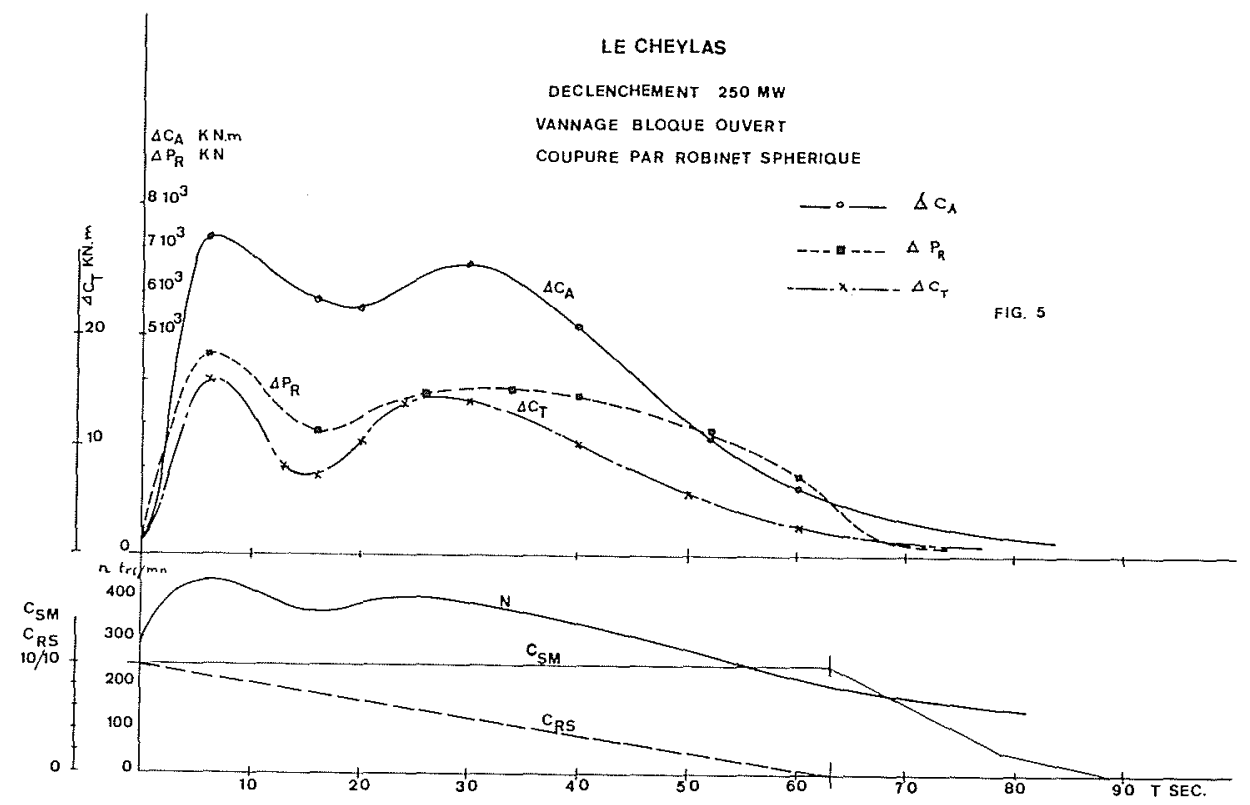

Figure 5 - Evolution des fluctuations des paramètres de la figure 3, en fonction de la vitesse de rotation, avec vannage bloqué à pleine ouverture durant la manœuvre de fermeture du robinet sphérique. 


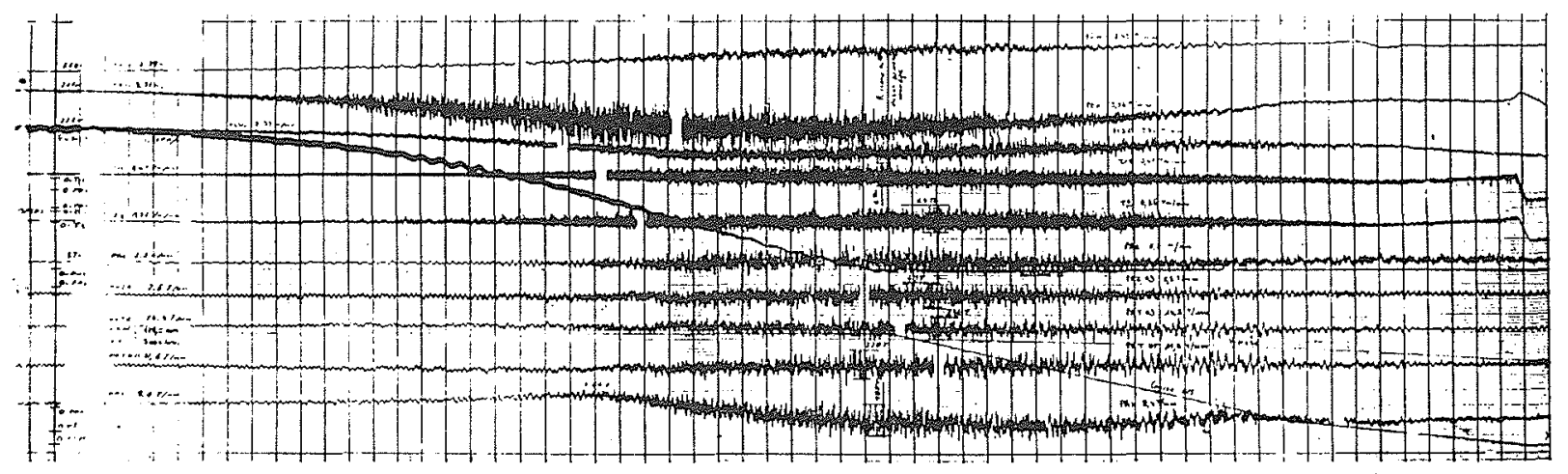

COUPURE EN CHARGE PAR RS $\mathrm{N}=250 \mathrm{MW}$

\section{LE CHEYLAS}

Figure 6 - Diagramme de coupure en charge par le robinet sphérique, le distributeur étant maintenu à pleine ouverture et le groupe découplé du réseau à puissance nulle.

et les poussées radiales sur le palier turbine sont de $\pm 230 \mathrm{~T}$. La poussée axiale moyenne s'élève à $200 \mathrm{~T}$ avec des fluctuations de $\pm 150 \mathrm{~T}$. On retrouve les mêmes fréquences caractéristiques que pour les essais précédents.

\section{Coupure en charge par le robinet sphérique}

Durant cet essai, le groupe reste couplé au réseau, le distributeur étant maintenu à pleine ouverture, la fermeture en charge est assurée par le robinet sphérique, et le groupe est découplé du réseau à puissance nulle. La figure 6 reproduit l'enregistrement de cet essai au cours duquel les fluctuations de couple sur les directrices et de poussées radiales sont restées inférieures à celles mesurées lors des déclenchements. On remarque, par contre, des fluctuations de pression dans la bâche, à l'aval du robinet sphérique atteignant $180 \mathrm{~m}$ alors qu'elles ne dépassent pas $60 \mathrm{~m}$ au déclenchement.

\section{Fonctionnement en pompe}

\section{Démarrage en pompe}

Comme nous l'avons vu, le démarrage en pompe s'effectue en asynchrone, roue dénoyée. Le couple asynchrone possède une composante pulsatoire dont la fréquence varie de $100 \mathrm{~Hz}$ à zéro durant la phase de mise en vitesse de la machine. Lorsque cette fréquence approche la fréquence propre de l'arbre, un phénomène de résonance se traduit par des fluctuations du couple de torsion, qui peuvent atteindre des valeurs élevées, en particulier si l'accélération du rotor est faible. L'importance de l'amplification se calcule avec une bonne précision si l'on connait l'amplitude des fluctuations du couple asynchrone.

On retrouve de nouveau des fluctuations de couple de $\pm 110 \mathrm{Tm}$ pendant la phase de barbotage après renoyage de la roue, avant l'ouverture du vannage.

Pendant ce fonctionnement de courte durée (environ 5 secondes), la poussée radiale atteint $\pm 130 \mathrm{~T}$ et les fluctuations de pression dans l'aspirateur s'élèvent à $\pm 26 \mathrm{~m}$.
Dès que le vannage commence à s'ouvrir, on observe une décroissance rapide des fluctuations, sauf en ce qui concerne les sollicitations des directrices qui atteignent un maximum de $\pm 1,7 \mathrm{Tm}$ au tiers de la course du servo-moteur. Cet accroissement des fluctuations du couple sur les directrices peut être la conséquence de plusieurs phénomènes :

- décollements instables sur les profils;

- recirculation dans la roue à débit partiel ;

- pointe de cavitation générale dans la roue, réagissant sur l'écoulement à la sortie.

Une visualisation de l'écoulement dans le distributeur pourrait permettre de mieux connaître l'origine du phénomène.

La figure 7 représente l'évolution des diverses fluctuations pendant la prise de débit en pompe. Leurs

LE CHEYLAS

PRISE DE DEBIT EN POMPE

FLUCTUATIONS $\mathrm{C} / \mathrm{C}$

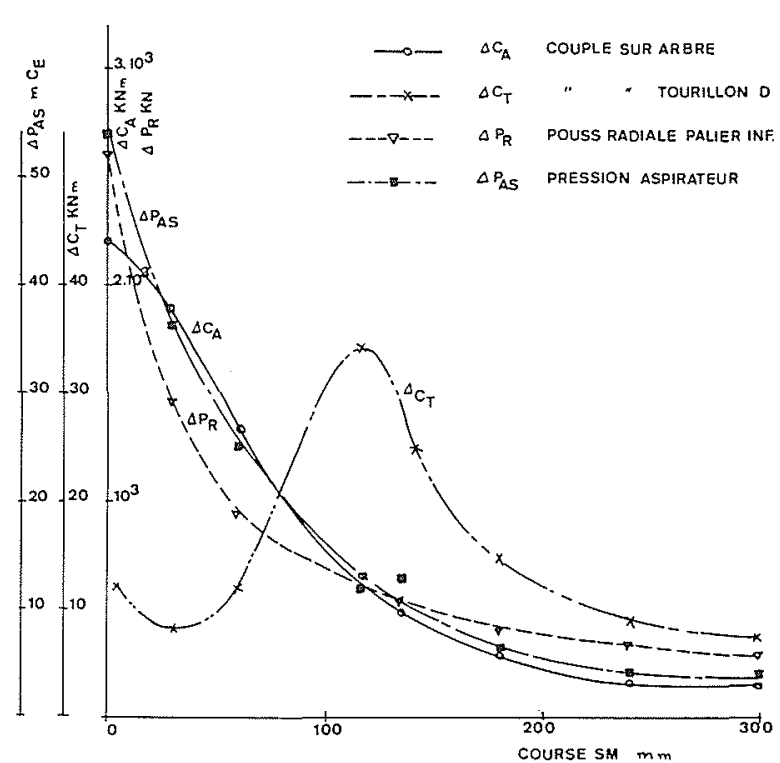

Figure 7 - Evolution des fluctuations des paramètres en fonction de l'ouverture du vannage pendant la prise ou débit en pompe. 


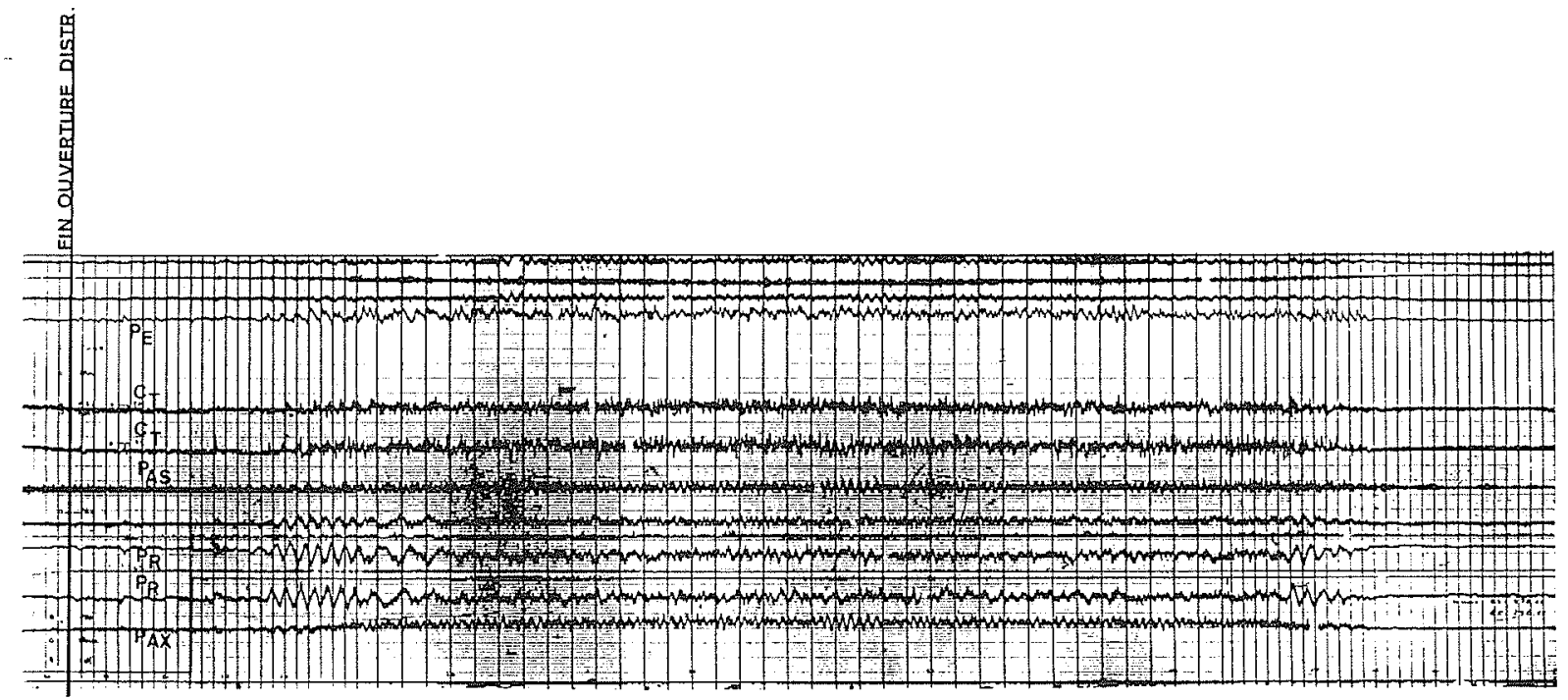

FONCTIONNEMENT AU VOISINAGE DU FESTON

\section{LE CHEYLAS}

Figure 8 - Diagramme de démarrage en pompe sous chûte élevée. Fonctionnement au voisinage de feston.

valeurs relevées en régime établi sont les suivantes: - $\pm 6,5 \mathrm{Tm}$ sur l'arbre du groupe, à la fréquence $f_{0}$; - $\pm 0,35 \mathrm{Tm}$ sur les tourillons de directrice, à la fréquence $f_{R}$;

$- \pm 12 T$ sur le palier turbine, à la fréquence $f_{0}$.

\section{Démarrage en pompe sous chute élevée}

A l'occasion d'un démarrage en pompe, alors que la chute était proche de sa valeur maximale, un phénomène d'instabilité s'est manifesté une vingtaine de secondes après la fin d'ouverture du vannage. L'enregistrement reproduit sur la figure 8 montre l'apparition brutale de fluctuations importantes. On note en particulier des poussées radiales de $\pm 150 \mathrm{~T}$ sur le palier turbine et des couples pulsatoires de $\pm 4,5 \mathrm{Tm}$ sur les tourillons de directrices. Ce phénomène, d'une durée de $60 \mathrm{sec}$. environ, s'explique par l'évolution de la cote du plan d'eau dans la cheminée d'équilibre, durant le régime transitoire consécutif à la prise de débit. La figure 9 représente la variation de la hauteur de refoulement en fonction du temps. Elle est tirée des mesures de pression effectuées par EDFDTG au pied de la cheminée d'équilibre et en différents points des adductions. Ces valeurs sont transposées sur le diagramme débit chute de la figure 10 . Le point A représente les conditions de fonctionnement permanent sous la chute maximale. Durant le régime transitoire, l'augmentation de la chute aux bornes de la machine se traduit par un déplacement du point de fonctionnement en $B$ et $C$ pour l'ouverture déterminée du diffuseur. La zone ombrée représente la variation de chute correspondant aux fluc-

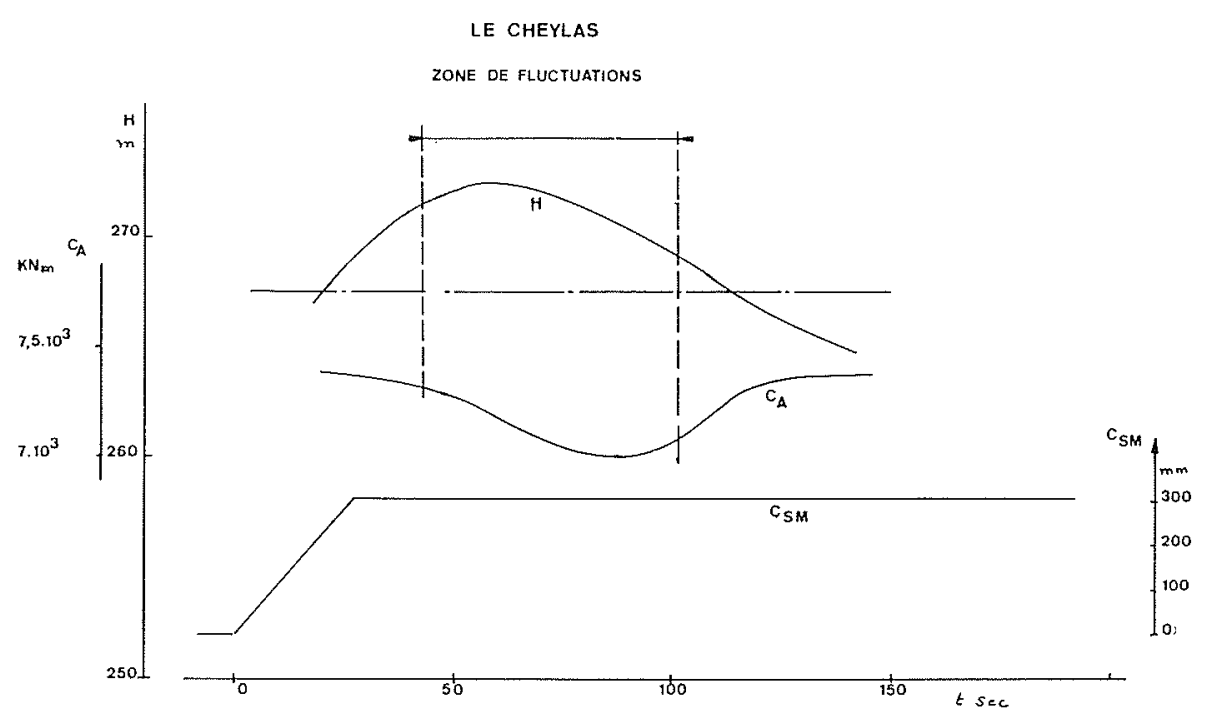

Figure 9 - Variation de la hauteur de refoulement en fonction du temps. 


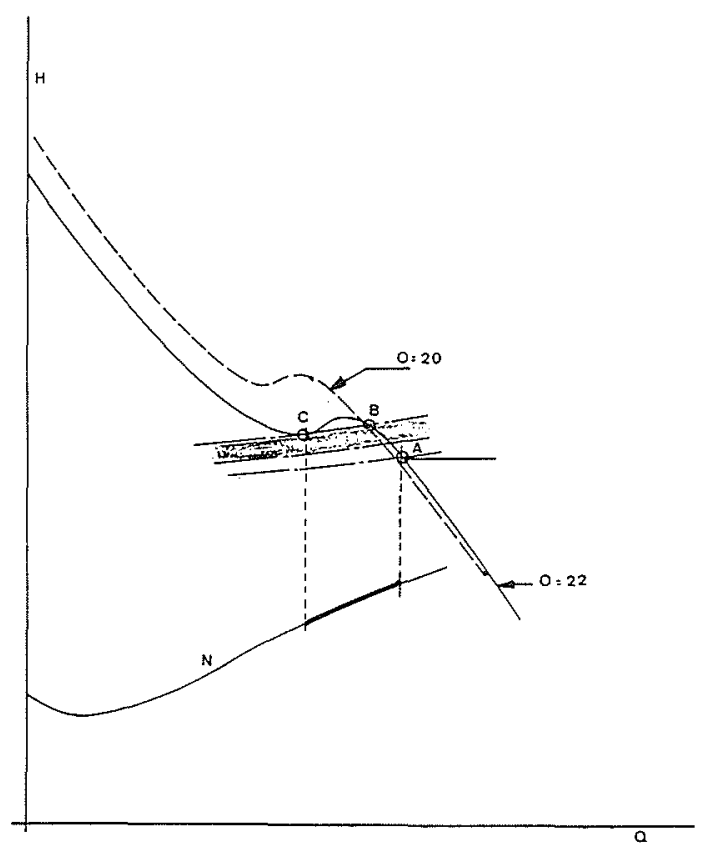

Figure 10 - Diagramme débit/chute au pied de la cheminée d'équilibre et en différents points d'adduction.

tuations importantes relevées sur la machine. L'apparition de ces perturbations s'explique par le fonctionnement momentané de la pompe au voisinage $\mathrm{du}$ feston. La variation du couple moteur représenté sur la figure 9 correspond à la variation de puissance absorbée par la pompe lorsque le point de fonctionnement se déplace de $A$ à $C$ le long de la caractéristique $Q-h$.

La machine travaillant sous la chute maximale peut entrer dans le "feston" pendant le régime transitoire. Des oscillations de débit apparaissent alors, puis se calment après étouffement du transitoire.
Bien que ce phénomène n'entraîne pas de danger pour la machine, il a été complètement supprimé en adoptant une ouverture en pompe légèrement inférieure à l'ouverture initiale, afin d'éloigner la zone instable de la chute transitoire maximale.

\section{Déclenchement normal en pompe}

Les figures 11 et 12 représentent respectivement l'enregistrement d'un déclenchement en pompe et l'évolution des fluctuations des couples et de la poussée radiale. On remarque que les sollicitations diverses et les pulsations de pression les plus importantes apparaissent dans le quadrant pompe frein, peu après l'inversion du débit qui se produit en $5 \mathrm{sec}$. environ. La vitesse de rotation du groupe décroit de 300 à $20 \mathrm{tr} / \mathrm{min}$ en 21 secondes, temps de fermeture du vannage. Les plus grandes amplitudes de fluctuation de la poussée radiale atteignent $160 \mathrm{~T}$ et se produisent à une fréquence un peu supérieure à $2 \mathrm{~Hz}$. Les pulsations de couple sur l'arbre et les directrices s'élèvent respectivement à $\pm 90 \mathrm{Tm}$ et $\pm 4 \mathrm{Tm}$.

\section{Déclenchement en pompe vannage bloqué}

Trois essais ont été réalisés avec des ouvertures de vannage progressivement croissantes. La figure 13 se rapporte au déclenchement à l'ouverture nominale en pompe. Le débit et la vitesse de rotation s'inversent respectivement en 4 et 13 secondes. L'emballement en turbine est atteint 28 secondes après la disjonction. Notons qu'il s'agit là d'un emballement partiel, le robinet sphérique en cours de fermeture contrôlant déjà le débit. Les fluctuations enregistrées par tous les capteurs commencent un peu avant l'inversion de débit et croissent jusqu'à un maximum au voisinage de la vitesse nulle. Elles décroissent dans le quadrant turbine et passent par un nouveau maximum à l'emballement où se produisent les sollicita-

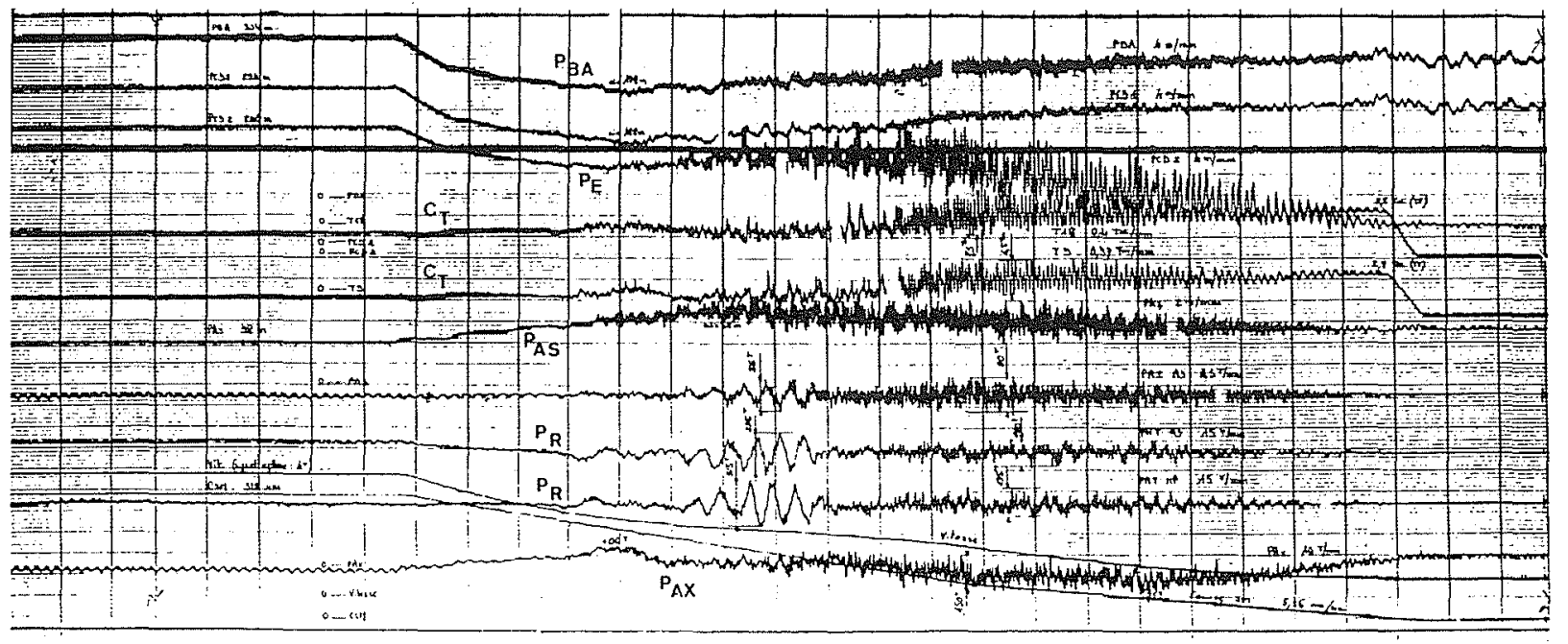

DECLENCHEMENT EN POMPE

LE CHEYLAS

Figure 11 - Diagramme d'un déclenchement en pompe. 


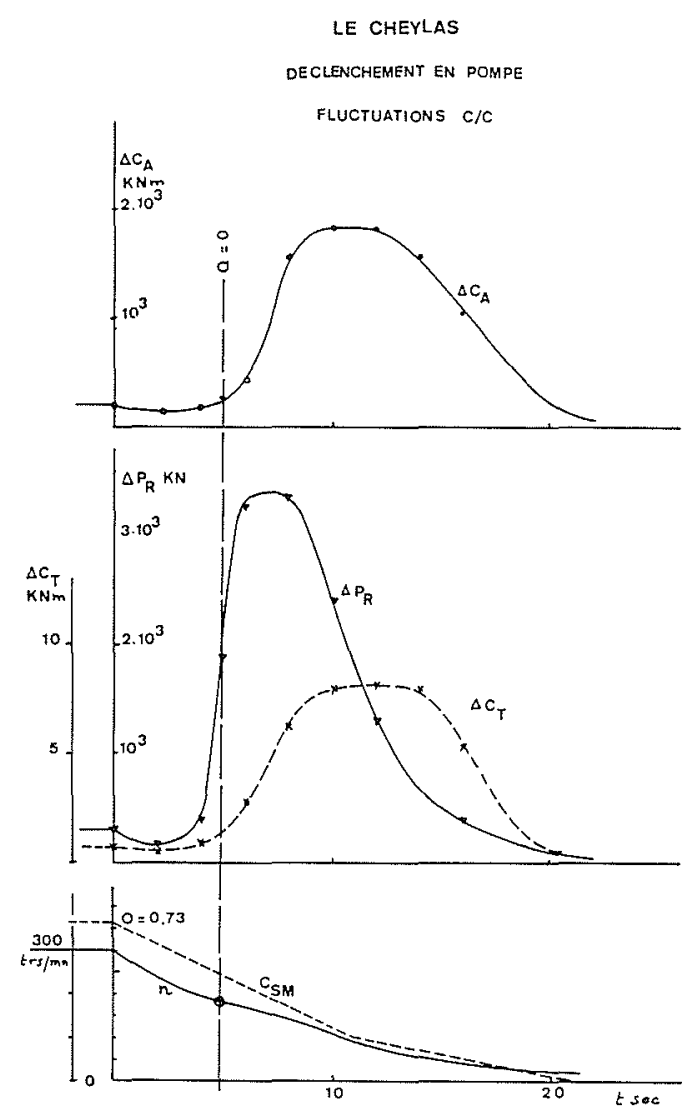

Figure 12 - Evolution des fluctuations des couples et de la poussée radiale lors d'un déclenchement en pompe.

tions les plus importantes de ce régime transitoire. Les valeurs extrêmes restent du même ordre de grandeur que celles relevées lors d'un déclenchement normal.

Les analyses spectrales effectuées sur des intervalles de temps de 2 secondes montrent que les fréquences des pulsations de couple sur les directrices et de poussée radiale restent proportionnelles à la vitesse instantanée de rotation.
Comparaison des résultats de mesures sur prototype et sur modèle

\section{Couples sur les directrices}

Nous comparons les valeurs réduites des pulsations de couple $C_{11}$ mesurées sur modèle et sur prototype industriel. Pour déterminer convenablement les valeurs réduites d'après les essais industriels, il importe de définir avec une bonne précision la chute instantanée aux bornes de la machine. Pour ce faire, nous utilisons les enregistrements magnétiques des pressions dans la bâche et l'aspirateur, qui sont restituées sur papier en filtrant le signal à $1 \mathrm{~Hz}$. Cette procédure permet d'éliminer les fluctuations et d'obtenir les valeurs moyennes des pressions à partir desquelles la chute instantanée est évaluée avec une marge d'erreur de $\pm 5 \mathrm{~m}$.

Les fluctuations de couple sur les tourillons à l'emballement en turbine sont tracées sur la figure 14.

Les figures 15 et 16 se rapportent aux fluctuations de couple mesurées lors des essais de déclenchement en pompe avec vannage bloqué à diverses ouvertures.

Cette comparaison met en évidence une bonne concordance entre les mesures in situ et en laboratoire.

\section{Poussées radiales}

La comparaison des résultats de mesure des poussées radiales sur modèle et sur prototype est plus délicate. Les difficultés d'étalonnage des dispositifs de mesures industrielles entraînent une imprécision difficilement chiffrable.

Nous donnons néanmoins sur la figure 17 les valeurs réduites des fluctuations de poussée à l'emballement $P_{11}$ tirées des essais industriels et sur modèle, en fonction de la vitesse d'emballement réduite. Nous notons, entre les deux résultats de mesure, des écarts plus importants que pour les couples sur les directrices.

Le système de mesure sur modèle réduit peut s'étudier de façon assez complète. Sa réponse dynamique

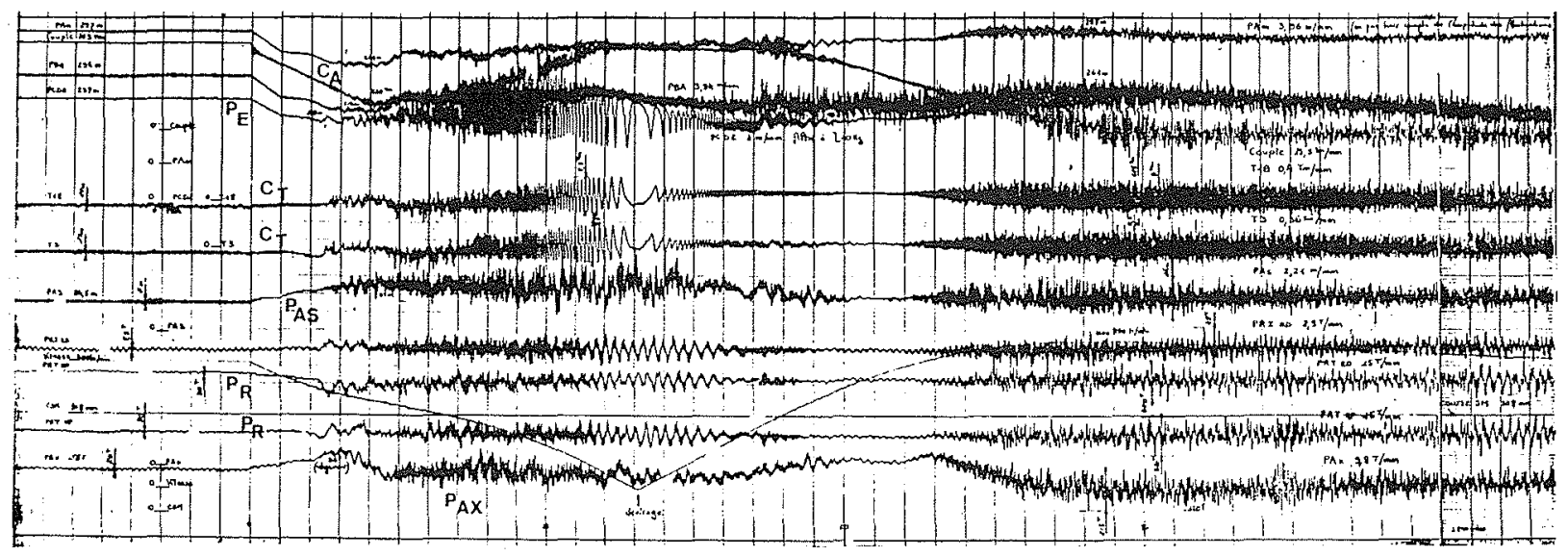

DECLENCHEMENT EN POMPE VANNAGe BLoQue

\section{LE CHEYLAS}

Figure 13 - Diagramme d'un déclenchement en pompe-vannage bloqué. 


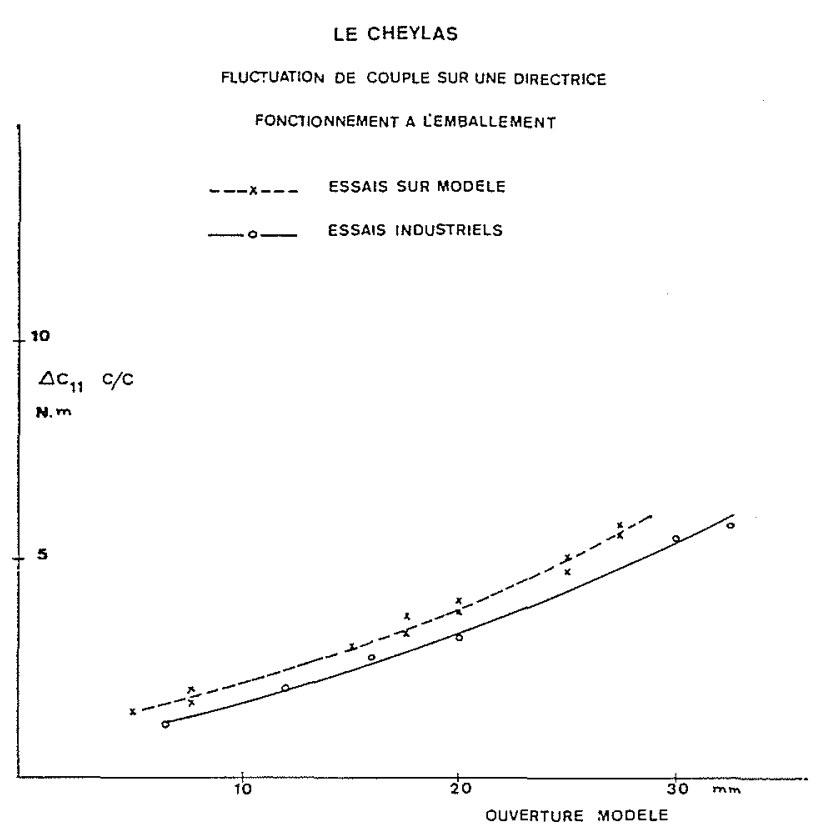

Figure 14 - Fluctuations du couple sur les tourillons d'une directrice. Fonctionnement à l'emballement. peut s'étalonner par la mesure d'un balourd connu. Par contre, sur la machine industrielle, l'étalonnage statique du palier et les conditions de mesure font que les résultats sont vraisemblablement moins précis que sur modèle. Il n'est cependant pas à rejeter qu'une différence existe entre modèle et prototype. Jusqu'à présent, les valeurs mesurées sur des machines industrielles restent inférieures à celles déduites des essais sur modèle.

\section{Conclusion}

Les mesures sur les machines industrielles de puissances élevées permettent d'accroître nos connaissances sur le fonctionnement des turbines-pompes, plus particulièrement lors des régimes transitoires où se manifestent des efforts pulsatoires à fréquences élevées pouvant provoquer une fatigue du matériel.

Le présent exposé soulève certaines questions concernant les aspects suivants :

\section{LE CHEYLAS \\ DEVIRAGE POMPE}

FLUCTUATION DE COUPLE SUR UNE DIRECTRICL
Figure 15 - Fluctuations du couple sur les tourillons d'une directrice. Déclenchement en pompe avec vannage bloqué à diverses ouvertures $(7,5$ et $8 \mathrm{~mm})$.

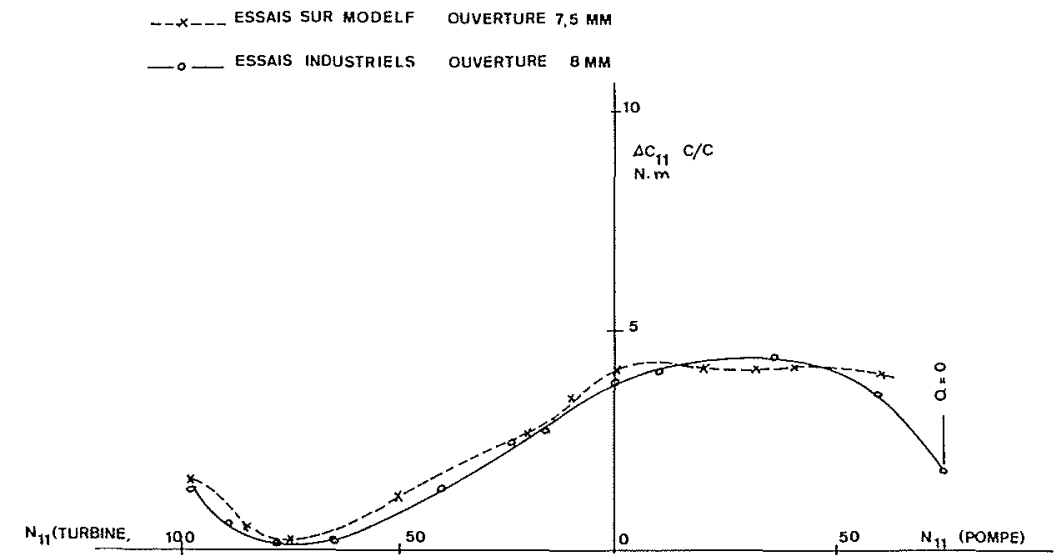

LE CHEYLAS

DEVIRAGE POMPE

ESSAIS SUR MODELE

OUVERTURE $22,5 \mathrm{~mm}$

_o- essais industriels

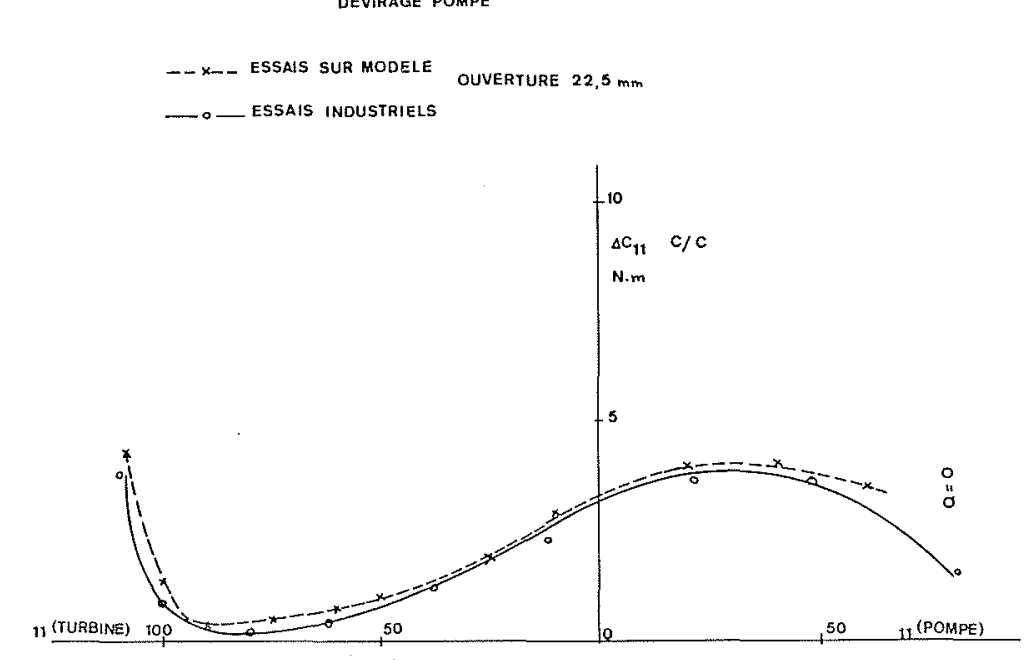

Fig. 16 - Fluctuations du couple sur les tourillons d'une directrice. Déclenchement en pompe avec vannage bloqué avec une ouverture de $22,5 \mathrm{~mm}$. 


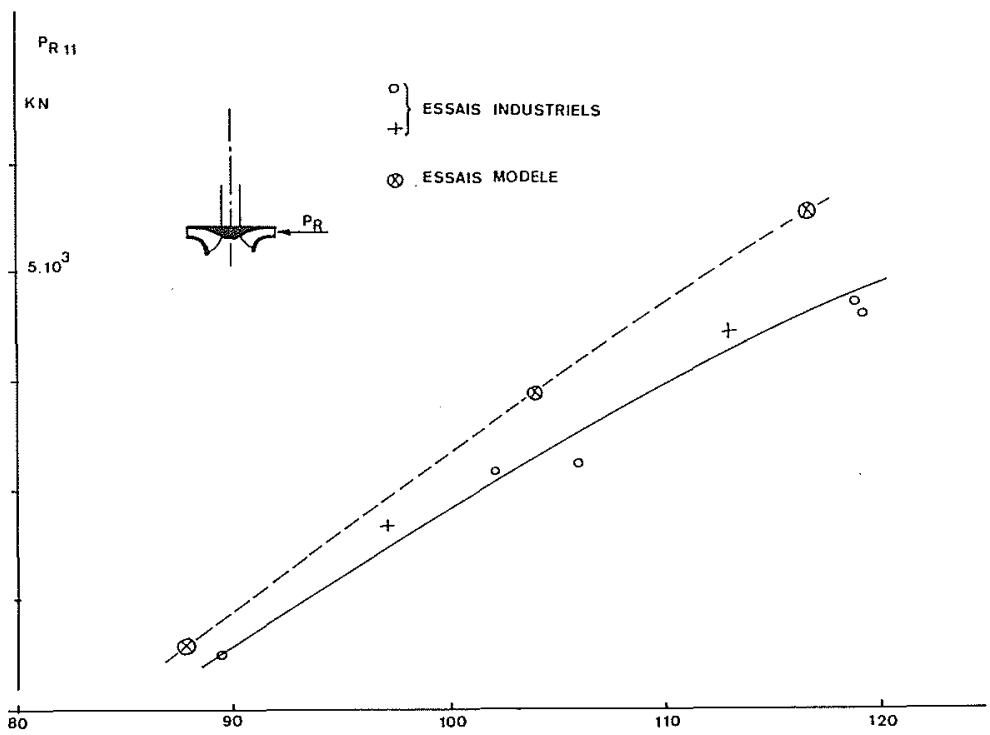

Fig. 17 - Valeurs réduites des fluctuations ou poussée radiales à l'emballement tirées des essais industriels et sur modèle.

\section{- pulsations du couple moteur}

En marche à vide ou lors des emballements de la machine, le couple moteur moyen est très faible. Les fluctuations importantes qui se manifestent lors de ces régimes se traduisent par des changements de sens du couple sur l'arbre. Il importe de tenir compte de ces sollicitations alternées pour la conception des accouplements. La fréquence propre de torsion de l'arbre doit être nettement supérieure à la fréquence de rotation correspondant à la survitesse maximale de la machine.

Des fluctuations à basse fréquence du couple moteur peuvent compromettre la stabilité du groupe en marche à vide. L'étude de ce problème nécessite une visualisation sur modèle réduit.

\section{- couple sur les directrices}

Les couples hydrodynamiques moyens restent en général faibles vis-à-vis des fluctuations. Il en ré- sulte des changements de sens du couple sur les directrices et les organes de vannage doivent être calculés en tenant compte des efforts alternés qu'ils ont à supporter. Un soin particulier doit être apporté au choix des jeux fonctionnels des tourillons et de l'embiellage de commande. Pour éviter les risques de corrosion de friction, des bronzes spéciaux sont utilisés pour la réalisation des douilles de guidage des tourillons.

\section{- poussées radiales}

Il est indispensable de réaliser des paliers de très grande rigidité pour supporter, sans déformation excessive, les poussées radiales élevées qui se manifestent pendant les régimes transitoires. Cette rigidité des supports est, par ailleurs, nécessaire pour obtenir une vitesse critique suffisamment élevée vis-à-vis de la survitesse maximale.

\section{Discussion}

Président : J. CHEVALIER

Le Président, félicite les auteurs pour la finesse des mesures effectuées et les comparaisons cntre essais industriels et essais sur modèle. Il se demande toutefois, à propos des trois communications qui seront présentées au cours de la séance quel est le degré de généralité de ce type d'étude.

A la suite des interventions de MM. le Professeur OSTERWALDER, GILMER, JAQUET et GUITON, des compléments d'information concernant les aspects hydrauliques sont fournis par M. JARRIAND.

Il y a une bonne concordance entre les caractéristiques hy- drauliques du modèle et celles de la machine industrielle y compris la position du feston de la courbe $H . Q$. I I faut toutefois noter que le distributeur est réglé sur le site ce qui permet d'ajuster les courbes. H.Q., mais modifie un peu la puissance.

La similitude de Mach qu'on pourrait souhaiter pour des phénomènes où il existe des figures de cavitation très développées ne peut pas être respectée sur le modèle.

Après avoir constaté la bonne concordance des essais dynamiques sur modèle MM. DODU et LIESS demandent des précisions sur les conditions de similitude dynamique. 
Il n'y a pas de similitude hydroélastique répond M. JARRIAND, mais la fréquence propre des directrices est très élevée par rapport aux fréquences d'excitation. Les paliers sont différents sur le modèle mais ils sont très rigides et l'amortissement plus faible. Les poussées radiales diffèrent de $20 \%$ à l'emballement.

Il semble difficile d'apporter une réponse à la question de M. GUILloud qui demande s'il est possible d'expliquer l'apparition de la fréquence de $2 \mathrm{~Hz}$ dans les mesures de fluctuations de poussées radiales en fonctionnement en pompe-frein. Il n'existe pas de fréquence propre du système à $2 \mathrm{~Hz}$, mais MM. JARRIAND et ANDRE (DTG) ont déjà trouvé cette fréquence sur des turbines Francis.

Les participants s'accordent à dire que le problème des fluctuations de poussée radiale est moins aigu dans le cas des turbines-pompes multicellulaires.

M. JARRIAND confirme, à la suite d'une question de
M. WEGNER, que c'est à l'emballement que la machine est la plus sollicitée.

M. ROVARO présente les essais que la Société HYDROART a pu effectuer sur trois des machines réversibles multicellulaires du site de Chiotas Piastra. Les essais en circuit fermé (une machine en pompe, une autre en turbine... etc.) ont permis de faire des mesures hydrauliques et dynamiques précises dans tous les régimes de fonctionnement intéressants. Les valeurs maximales de vibrations obtenues sur le palier inférieur lors des transitions sont de l'ordre de 100 à $150 \mu$ crête à crête.

Sur l'intervention de M. AMBLARD, M, ROVARO précise qu'il y a une bonne concordance entre les caractéristiques du modèle et celles de la machine industrielle mais que la puissance à débit nul obtenue sur la machine industrielle est un peu plus faible que celle obtenue sur le modèle et qu'il y a peu de différences entre les machines du site. 